\title{
Is delayed foveal feedback critical for extra-foveal perception?
}

\author{
Christopher D. Chambers ${ }^{1 \dagger}$ \\ Christopher P. G. Allen ${ }^{1}$ \\ Leah Maizey $^{1}$ \\ Mark A Williams ${ }^{2}$
}

1. Cardiff University Brain Research Imaging Centre (CUBRIC), School of Psychology, Cardiff University, Park Place, CF10 3AT, United Kingdom

2. Centre for Cognition and its Disorders, Macquarie University, Sydney, New South Wales, 2109, Australia

\footnotetext{
$\uparrow$ Corresponding author

Christopher D. Chambers

School of Psychology

Cardiff University

CF10 3AT

United Kingdom
}

Email: chambersc1@cardiff.ac.uk

Keywords: human vision; transcranial magnetic stimulation; feedback

Full Reference: Chambers, C.D., Allen, C.P.G., Maizey, L. \& Williams, M.A. (2013). Is delayed foveal feedback critical for extra-foveal perception? Cortex, 49, 327-335. 


\begin{abstract}
Recent neuroimaging evidence suggests that visual inputs arising beyond the fovea can be 'fed back' to foveal visual cortex to construct a new retinotopic representation. However, whether these representations are critical for extra-foveal perception remains unclear. Using transcranial magnetic stimulation we found that relatively late $(350-400 \mathrm{msec})$ disruption of foveal retinotopic cortex impaired perceptual discrimination of objects in the periphery. These results are consistent with the hypothesis that feedback to the foveal retinotopic cortex is crucial for extra-foveal perception, and provide additional evidence for 'constructive' feedback in human vision.
\end{abstract}




\section{Introduction}

Feedback is a ubiquitous concept in cognitive neuroscience, being implicated as the engine of attentional selection (Desimone and Duncan, 1995; Duncan et al., 1997; Kastner and Ungerleider, 2000), mental imagery (Kosslyn et al., 2001; Kosslyn et al., 1999) and visual awareness (Lamme, et al., 1998; Ro et al., 2003). Established accounts of feedback emphasise top-down modulation of pre-activated feedforward representations (Ress and Heeger, 2003; Super et al., 2001). Recently, however, multivoxel pattern analysis of fMRI data has provided evidence that peripheral object information can be decoded from visual cortical regions that represent central foveal space (Williams et al., 2008). These data point to the existence of a feedback mechanism that does more than simply modulate existing activity: it constructs a new representation.

Although this previous fMRI study is suggestive of a 'constructive' feedback mechanism (Williams, et al., 2008), definitive evidence remains lacking. In particular, it is not known whether peripheral object information in the foveal retinotopic cortex is causally constitutive in shaping perception, or whether such activity is a redundant by-product of activity elsewhere. Furthermore, the temporal resolution of the BOLD measurement is too low to definitively distinguish a foveal feedback hypothesis from several competing explanations, including cross-activation via horizontal cortical connections (Gilbert, 1993; Lamme, Super, et al., 1998) and hemodynamic point spread (Arthurs and Boniface, 2003; Logothetis, 2008).

We contrasted these accounts in the present study using the complementary approach of transcranial magnetic stimulation (TMS) together with an almost identical behavioural task to Williams et al. (2008). If foveal visual cortex is crucial for processing extra-foveal stimuli then disrupting it should impair the ability to discriminate peripheral visual objects. Moreover, if peripheral discrimination requires foveal feedback, then the critical timecourse of behavioural effects should be substantially delayed relative to the well-established feedforward timing of the visual system ( 40-120msec; e.g. Amassian et al., 1989; Corthout et al., 1999; Kammer et al., 2005; Lamme and Roelfsema, 2000). In Experiment 1 we tested these hypotheses by applying TMS over the posterior termination of the calcarine sulcus between $-150 \mathrm{~ms}$ and $+500 \mathrm{~ms}$ relative to stimulus onset. To anticipate, TMS significantly impaired discrimination accuracy when given 350-400ms after stimulus onset. In Experiment 2 we confirmed that calcarine TMS disrupted early processing of foveal stimuli, and we also tested whether late disruption of visual perception was specific for peripheral stimuli. 


\section{Methods}

\subsection{Experiment 1}

\subsubsection{Participants}

Eighteen neurologically healthy, right-handed volunteers were recruited in Experiment 1 (9 females; mean age $=26.2$ years). All participants had normal or corrected-to-normal vision and were initially screened for medical contraindications to TMS and MRI. The study was approved by the Human Research Ethics Committee at the School of Psychology, Cardiff University.

\subsubsection{Apparatus}

Experimental sessions were conducted in a darkened laboratory. Visual stimuli were presented at a mid-sagittal viewing distance of $65 \mathrm{~cm}$, on a 21 -inch CRT monitor $(60 \mathrm{~Hz}$ vertical refresh rate; $1024 \times 768$ resolution; black background). Throughout the experiment, the participant's head was fixed within a chinrest. Eye gaze was monitored on all trials with a Cambridge Research Systems video eye-tracking system, mounted in the chin rest $(250 \mathrm{~Hz}$ sampling rate). Trials in which gaze deviated more than $2^{\circ}$ from fixation or in which eyeblinks occurred were discarded. These criteria resulted in the exclusion of $1.1 \%$ of trials due to blinks, and $0.1 \%$ of trials due to saccades. The total percent of eye errors did not differ significantly between TMS sites $(1.14 \% ; t(16)=0.67, p=.51)$.

\subsubsection{TMS and MRI parameters}

Cortical stimulation was administered with a Magstim Super Rapid ${ }^{2}$ system using a figure-ofeight coil $(70 \mathrm{~mm})$ with the handle oriented upright to induce current flow along the superiorinferior axis. The TMS coil was fixed in position using a clamp and tripod and participants wore foam earplugs throughout the experiment.

In different sessions, TMS was applied to the posterior termination of the calcarine sulcus (the foveal site) or to an occipital control region (the non-foveal site; see Figure 1). On each trial, a double-pulse of TMS was applied at one of seven possible times relative to target onset ($150 /-100,-50 / 0,+50 / 100,+150 / 200,+250 / 300,+350 / 400,+450 / 500 \mathrm{msec})$, at either a high 'effective' intensity or a low 'ineffective' intensity (120\% or $40 \%$ motor threshold obtained via a single-pulse protocol, as described previously; Ruff et al., 2006; Schenkluhn et al., 2008; Varnava et al., 2011). Mean stimulator output for low and high intensities was $20 \%$ and $58 \%$, 
respectively. An initial test at the start of each session confirmed that the high intensity TMS did not elicit phosphenes.

[Insert Figure 1 about here]

To control for spatial priming effects we also delivered TMS prior to target onset. Our rationale for doing so was that activation of foveal retinotopic space (via TMS) might cue attention away from peripheral stimuli, providing an obvious explanation for any perceptual deficit. To address this issue, TMS was applied from $150 \mathrm{msec}$ before target onset, when any such attentional priming effects should be maximal (Müller and Rabbitt, 1989).

Prior to testing, structural MRI scans of each participant were acquired in a 3T whole-body GE scanner. Target sites for TMS were localised in each participant's scan using MRIcro imaging software (Rorden and Brett, 2000). Neuroanatomical definitions for each site were based on occipital sulcal landmarks. The foveal (posterior calcarine or 'p-calcarine') site was defined as the hemispheric mid-point between the posterior termination of the left and right calcarine sulci. The non-foveal (non-calcarine control) site was referenced to a location $\sim 15 \mathrm{~mm}$ superior to the p-calcarine site, beyond the termination of the calcarine sulci. On average, the non-calcarine site was localised $15.7 \mathrm{~mm}$ rostral to the foveal site on the cortical surface $(S D=2.4 \mathrm{~mm})$ and $16.8 \mathrm{~mm}$ rostral on the scalp surface $(S D=2.3 \mathrm{~mm})$. The mean scalp-cortex distance for the p-calcarine $(M=12.4 \mathrm{~mm}, S D=2.6 \mathrm{~mm})$ and non-calcarine sites $(M=11.6 \mathrm{~mm}, S D=1.9 \mathrm{~mm})$ did not differ significantly $[t(16)=1.4, p=.19]$. At the beginning of each testing session, one region of interest was coregistered to the scalp surface using MR coregistration software (MRIReg) and a magnetic tracking device (miniBIRD 500, Ascension Tech).

\subsubsection{Stimuli and Procedure}

In the discrimination task (Figure 1a), participants decided whether two 'spiky' stimuli presented in opposite diagonal locations were the same or different. Participants responded with the index or middle finger of their right hand to indicate a 'same' or 'different' response, respectively. Participants were instructed to maintain fixation throughout each trial and respond as accurately as possible, with no emphasis on speed. 
Target stimuli were selected randomly from a set of 1296 pre-generated 'spiky' stimuli, which vary by six levels along four dimensions (Op de Beeck et al., 2006). Prior to receiving TMS, the duration of the target stimulus was titrated in each participant to yield 70-75\% discriminations $\left(d^{\prime}=1.0-1.5\right)$, with the constraint that the duration could not exceed $150 \mathrm{msec}(M=78 \mathrm{msec}, S D=39 \mathrm{msec})$. If a participant could not perform at a sufficient accuracy level for the longest duration, then the titration was repeated using a more constrained set of spiky stimuli in which the minimal difference between stimuli on 'different' trials was increased. On average, participants achieved 74.4\% $\left(d^{\prime}=1.40\right)$ performance during low-intensity TMS of foveal and non-foveal sites.

Each trial was self-initiated by button press and commenced with the onset of the central fixation stimulus (grey square, $0.15^{\circ} \times 0.15^{\circ}, 500 \mathrm{msec}$ ). The target array was then presented, which consisted of the two 'spiky' stimuli in opposite diagonal locations $\left(1.85^{\circ} \mathrm{H} \times 1.65^{\circ} \mathrm{W}\right.$, each at $7^{\circ}$ eccentricity). An indefinite response window was then followed by a fixed intertrial interval of $750 \mathrm{msec}$, after which the participant could initiate the next trial via button press.

The diagonal configuration of spiky stimuli was held constant throughout each block of trials, and alternated between blocks. Each block contained 116 trials, including four initial 'practice' trials (not analysed), followed by 112 experimental trials. Within each block containing TMS, four trials were presented per level of TMS intensity (low, high), TMS onset time relative to target onset $(-150 /-100,-50 / 0,+50 / 100,+150 / 200,+250 / 300,+350 / 400$, $+450 / 500$ ), and target type (same, different). Participants completed a total of eight such blocks per TMS site (p-calcarine, non-calcarine), thus yielding a total of 64 trials per TMS intensity, TMS onset time, and TMS site. The order of stimulation sites was blocked between sessions and counterbalanced across participants, with at least 24 hours between subsequent sessions.

Prior to receiving TMS, participants initially completed the same task during 'Sham' TMS, in which the coil was aligned perpendicular to the occipital scalp. The Sham condition was undertaken with the same parameters as the active sites, and simulated some of the sensory artefacts that accompany real TMS. These artefacts can distract or alert participants independently of cortical stimulation (Sawaki et al., 1999). Participants completed a total of eight Sham blocks prior to active TMS, divided between sessions for each of the TMS sites. 
Following completion of the experiment, the Sham blocks were compiled into a baseline that included the same number of trials per TMS intensity and TMS onset time as the active sites.

\subsubsection{Data Analysis}

The principal dependent variable was the $d^{\prime}$ of target discrimination. The hit rate (HR) was defined as the proportion of correct 'same' responses on 'same' trials, while the false-alarm rate (FAR) was defined as the proportion of 'same' responses on 'different' trials. To provide a full account of the results, we undertook a combination of linear and non-linear analyses.

\subsubsection{Non-linear regression analyses}

Based on previous studies that have revealed at least two critical periods of processing in visual cortex (e.g. Camprodon et al., 2010; Corthout et al., 1999; de Graaf et al., 2011; Heinen et al., 2005; Koivisto et al., 2010; Laycock et al., 2007; Silvanto et al., 2005a; Stevens et al., 2009), we modelled individual data using a double inverted Gaussian function, which permits up to two distinct TMS-induced epochs after target onset. This approach has the advantage of detecting both early and late effects of TMS on performance and can also capture individual differences in the critical timecourse of visual processing. A similar analytic approach to considering event-related TMS data was recently reported by Stevens et al. (2009).

Specifically, the following Gaussian model was fit to $d^{\prime}$ values separately in each participant at each level of TMS site (p-calcarine, non-calcarine, sham) and TMS intensity (low, high).

$$
y=y_{0}+a_{1} e^{\left[-0.5\left(\frac{x-x_{1}}{b_{1}}\right)^{2}\right]}+a_{2} e^{\left[-0.5\left(\frac{x-x_{2}}{b_{2}}\right)^{2}\right]}
$$

where $y_{0}=$ the intercept of the model,

$a_{1}, a_{2}=$ peak amplitude (change in $d^{\prime}$ from $y_{0}$ ) for the first and second periods, respectively;

$x_{1}, x_{2}=$ time of the first and second peaks; and

$b_{1}, b_{2}=$ full width half maximum (FWHM) bandwith of the first and second periods. 
The values of each parameter in each participant were permitted to vary freely in each model according to the following data-driven (1-3) and a priori (4-7) constraints:

1) $\quad\left(d^{\prime}{ }_{\max } \times-1\right)<y_{0}<d^{\prime}{ }_{\max }$

2) $\quad\left(d^{\prime}{ }_{\max } \times-1\right)<a_{1}<d^{\prime}{ }_{\max }$

3) $\quad\left(d^{\prime}{ }_{\text {max }} \times-1\right)<a_{2}<d^{\prime}{ }_{\text {max }}$

4) $-200<x_{1}<550$ (limited to $\pm 50 \mathrm{msec}$ beyond the range of TMS onset times)

5) $\quad-200<x_{2}<550$

6) $5<b_{1}<200$

7) $5<b_{2}<200$

where $d^{\prime}{ }_{\max }=$ the maximum $d^{\prime}$ value per participant for the specific combination of TMS site and TMS intensity.

According to this modelling approach, an influence of TMS on performance is denoted by the values of $a_{1}$ (first period) and $a_{2}$ (second period) to which $t$-tests can be applied. If these differed significantly from zero in a group analysis then the secondary parameters were also considered $\left(x_{1}, x_{2}, b_{1}, b_{2}\right)$.

\subsubsection{Linear analyses}

In addition to the non-linear regressions, we also undertook more conventional ANOVA analyses. Two-way repeated-measures ANOVAs including factors of TMS site (p-calcarine, non-calcarine, sham) and TMS onset time $(-150 /-100,-50 / 0,+50 / 100,+150 / 200,+250 / 300$, $+350 / 400,+450 / 500$ ) were undertaken separately for trials involving low or high intensity TMS, and for $d$ ' and response bias ( $c$ : the overall tendency to respond 'same' or 'different' independently of discrimination sensitivity). Where appropriate, analyses of simple main effects were corrected for multiple comparisons using the Holm-Bonferroni procedure (Aickin and Gensler, 1996).

\subsection{Experiment 2}

\subsubsection{Participants}


Ten volunteers were recruited in Experiment 2 ( 6 females; mean age $=23.9$ years $)$, one of whom had also participated in Experiment 1.

\subsubsection{Apparatus}

Equipment for visual presentation and eye-tracking was identical to Experiment 1. Trials in which gaze deviated more than $2^{\circ}$ from fixation or in which eye-blinks occurred were discarded, resulting in the exclusion of $2.0 \%$ of trials due to blinks and $1.0 \%$ of trials due to saccades.

\subsubsection{TMS and MRI Parameters}

General TMS and MRI methods were identical to Experiment 1, with the exception that TMS was now applied to the p-calcarine site only. On each trial, a double-pulse of TMS was delivered at one of two possible times relative to target onset $(+50 / 100$ or $+350 / 400 \mathrm{msec})$ at a high or low intensity ( $120 \%$ or $40 \%$ MT). Mean stimulator output for low and high intensities was $19 \%$ and $58 \%$, respectively.

\subsubsection{Stimuli and Procedure}

Participants completed the same task as in Experiment 1, but including blocks requiring discrimination of foveal or peripheral stimuli. All stimulus characteristics for peripheral targets were identical to Experiment 1. For foveal targets, the stimulus height and width were reduced in general accordance with the foveal vs. peripheral $\left(7^{\circ}\right)$ cortical magnification factor (Duncan and Boynton, 2003; $0.5^{\circ} \mathrm{H} \times 0.4^{\circ} \mathrm{W}$ ) and centred $0.5^{\circ}$ from fixation along the same diagonal vectors as the peripheral targets.

Prior to receiving TMS, the duration of the target stimulus was titrated in each participant to achieve $70-75 \%$ discriminations $(M=83 \mathrm{msec}, S D=16 \mathrm{msec})$. To ensure comparability of TMS effects, the duration of foveal and peripheral targets was matched within each participant. On trials with low-intensity TMS, average discrimination accuracy was $71.3 \%$ for foveal targets $\left(d^{\prime}=1.2\right)$ and $69.6 \%$ for peripheral targets $\left(d^{\prime}=1.1\right)$. These durations did not differ significantly $\left[t(9)=0.71, p=.50\right.$ for $\%$ correct; $t(9)=0.81, p=.44$ for $\left.d^{\prime}\right]$.

As in Experiment 1, the diagonal configuration of targets was held constant within blocks and alternated between blocks, with the order of alternation counterbalanced across participants. Each block contained 68 trials, including four initial 'practice' trials (not analysed) followed 
by 64 experimental trials. Participants completed four blocks each with foveal or peripheral stimuli. Each set of four blocks was presented in a continuous sequence, with the order (foveal-peripheral or peripheral-foveal) counterbalanced across participants. In total, 64 trials were obtained per participant at each level of Target location (foveal, peripheral), TMS intensity (low, high) and TMS onset time (+50/100, $+350 / 400 \mathrm{msec})$.

Following the main experiment, participants completed an additional phosphene test with the coil held in the same position over the p-calcarine site. During this procedure, participants were blindfolded and single pulse TMS was systematically increased in intensity until either the phosphene threshold was reached, the TMS became uncomfortable, or the intensity reached $100 \%$ stimulator output. If phosphene threshold was reached, the participant was then asked to report the location and approximate size of the percept.

\subsubsection{Data Analysis}

Three-way repeated-measures ANOVAs were undertaken for $d^{\prime}$ and $c$, including factors of Target location (foveal, peripheral), TMS intensity (low, high) and TMS onset time (+50/100, $+350 / 400)$.

\section{Results}

\subsection{Experiment 1}

Trials in which participants responded prior to TMS were excluded from analysis $(<0.1 \%)$, and the results for one participant were excluded entirely due to overall chance-level performance during low-intensity TMS. The outcome of the non-linear regression analysis is reported in Table 1 and Figure 2a. The models accounted for $83-92 \%$ of the variance in each sub-condition of TMS Intensity (low, high) and TMS site (p-calcarine, non-calcarine, sham). As shown in Table 1, the average peak amplitude ( $a$ : the change in $d^{\prime}$ ) differed significantly from zero only when high-intensity TMS was applied to the p-calcarine site at the later of the two possible Gaussian phases [parameter $a_{2}: t(16)=-3.43, p=.003$ ]. On average, TMS in this condition reduced $d^{\prime}$ by $0.69(S E=0.20)$ relative to the condition-specific intercept $(y 0=$ $1.44, S E=0.08$ ). This drop in $d^{\prime}$ was observed in $15 / 17$ participants and corresponds to a TMS-induced reduction in discrimination sensitivity of $46.9 \%$. The average time of this peak for the p-calcarine site was $332.2 \mathrm{msec}$ after target onset $(S E=20.5)$ with an average bandwidth $(\mathrm{FWHM})$ of $59.8 \mathrm{msec}(S E=16.5)$. Site-specific comparisons between the average modelled $d^{\prime}$ at each time point revealed similar effects (see Figure 2a). Additional paired $t$ - 
tests showed that $a_{2}$ for the p-calcarine high-intensity TMS trials was significantly reduced relative to $a_{2}$ for the corresponding low-intensity TMS trials $[M=0.15, S E=0.25 ; t(16)=2.4$, $p=.029]$, and was also reduced relative to the high-intensity TMS trials for both the noncalcarine control site $[M=0.21, S E=0.30 ; t(16)=2.5, p=.022]$ and the sham control condition $[M=0.02, S E=0.24 ; t(16)=2.1, p=.048]$.

\section{[Insert Table 1 about here]}

Conventional linear analyses produced a similar outcome. A two-way ANOVA of mean $d^{\prime}$ on high-intensity TMS trials, including factors of TMS site (p-calcarine, non-calcarine, sham) and TMS onset time $(-150 /-100,-50 / 0,+50 / 100,+150 / 200,+250 / 300,+350 / 400,+450 / 500)$ revealed no significant main effects (both $p>.16$ ) but did uncover a significant two-way interaction of TMS site $\times$ TMS onset time $[F(12,192)=1.81, p=.049]$. As shown in Figure $2 \mathrm{~b}$, analysis of simple main effects (Holm-Bonferroni corrected) revealed that stimulation of the p-calcarine site at $+350 / 400 \mathrm{msec}\left(d^{\prime}=1.05\right)$ reliably impaired performance relative to both the non-calcarine control site $\left(d^{\prime}=1.43 ; p=.004\right)$ and the sham control condition $\left(d^{\prime}=\right.$ $1.52 ; p=.002)$. This impairment in performance during $\mathrm{p}$-calcarine TMS was consistently observed across participants (Figure 2c).

[Insert Figure 2 about here]

In contrast, detection sensitivity at $+350 / 400 \mathrm{msec}$ did not differ significantly between the sham and non-calcarine control conditions $(p=.48)$, and no significant differences between $\mathrm{p}$ calcarine, non-calcarine and sham conditions were observed at any other TMS onset times. An additional paired $t$-test indicated that performance at $+350 / 400 \mathrm{msec}$ for the calcarine site $\left(d^{\prime}=1.05\right)$ differed significantly from the average $d^{\prime}$ of the six remaining calcarine TMS onset times $\left(d^{\prime}=1.28 ; t(16)=-2.54, p=.022\right)$. The corresponding two-way ANOVA of $d^{\prime}$ on low-intensity TMS trials revealed no significant interaction or main effects (all $p>.11$ ) and was therefore not considered further. However, at $+350 / 400 \mathrm{msec}$, calcarine TMS did significantly reduce $d^{\prime}$ on high-intensity TMS trials $\left(d^{\prime}=1.05\right)$ compared with the corresponding low-intensity TMS trials $\left(d^{\prime}=1.34 ; t(16)=-2.68, p=.016\right)$.

To what extent is the time-specific reduction in performance during p-calcarine stimulation associated with changes in response bias, that is, the tendency to judge the targets as 'same' or 
'different'? To answer this question we conducted the same two-way ANOVAs as above but on the $c$ parameter extracted from signal detection analysis (Figure 2d). For low-intensity TMS trials, no significant interaction or main effects were observed (all $p>0.1$ ). For highintensity TMS trials, no significant interaction $[F(12,192)=0.34, p=.98]$ or main effect of TMS time was detected $[F(6,96)=1.94, p=.08]$. However, now a significant main effect of TMS site was observed $[F(2,32)=4.30, p=.029]$, driven by significantly higher $c$ (tendency to respond 'different') on trials with calcarine $(M=-0.07, S E=.05)$ or non-calcarine TMS ( $M$ $=-0.1, S E=.04)$ compared with the sham control condition $(M=-0.16, S E=.05$; both $p<$ .016). The reason for this effect is not clear; it could potentially reflect a general influence of high-intensity active TMS on arousal, or perhaps a change in bias with practice (since sham blocks were always completed at the beginning of the p-calcarine and non-calcarine sessions). Importantly, however, since $c$ did not differ between the p-calcarine and non-calcarine sites $(p$ $=.49$ ), it cannot explain the site- or time- specific effects of p-calcarine TMS on $d^{\prime}$.

\subsection{Experiment 2}

Trials in which participants responded prior to TMS were excluded from analysis $(<0.1 \%)$. A three-way ANOVA of mean $d^{\prime}$, including factors of Target location (foveal, peripheral), TMS intensity (low, high) and TMS onset time $(+50 / 100,+350 / 400)$ revealed a significant threeway interaction $[F(1,9)=7.2, p=.025]$. Analysis of simple main effects revealed that effect of p-calcarine stimulation depended on target location and TMS onset time (see Figure 3). For foveal targets, high-intensity ( $v s$. low-intensity) TMS significantly impaired discrimination accuracy at $+50 / 100 \mathrm{msec}(p=.019)$ but not at $+350 / 400 \mathrm{msec}(p=.765)$. For peripheral targets, however, the opposite result was observed: consistent with Experiment 1, TMS impaired discrimination accuracy at $+350 / 400 \mathrm{msec}(p=.018)$ but not at $+50 / 100 \mathrm{~ms}(p=$ .398). The corresponding ANOVA of response bias (c) revealed no significant main effects or interactions (all $p>0.1$ ).

\section{[Insert Figure 3 about here]}

Following the main experiment, phosphenes were successfully elicited in 2 of 10 participants during single-pulse TMS of the p-calcarine site, at $81 \%$ and $84 \%$ stimulator output. In both cases the reported phosphene was foveal and did not overlap with the locations of the peripheral targets. These phosphene thresholds were substantially higher than the highintensity output for these participants in the main experiment ( $46 \%$ and $56 \%$, respectively). 
This was expected, as the TMS coil was deliberately oriented vertically in both experiments precisely to minimise the likelihood of phosphene induction, which typically most effective when the handle is positioned horizontally to induce current flow in a lateral-medial direction (e.g. Kammer et al., 2001).

\section{Discussion}

In Experiment 1 we found that TMS applied near the posterior (foveal) calcarine sulcus can disrupt the encoding of stimuli presented in the visual periphery, approximately $300-400 \mathrm{msec}$ after stimulus onset. In Experiment 2 we further observed that p-calcarine stimulation during the expected feedforward window (+50/100msec; Kammer et al., 2005a) selectively impaired discrimination of foveal but not peripheral targets; whereas stimulation during the later period $(+350 / 400 \mathrm{~ms})$ selectively impaired discrimination of peripheral but not foveal targets. The timecourse of this peripheral impairment appears too late to be explained by feedforward processing or horizontal cortical connections (Lamme and Roelfsema, 2000). Furthermore, the early impairment of foveal discrimination (combined with the foveal phosphene reports) indicates that p-calcarine TMS successfully disrupted foveal retinotopic cortex. Taken together, therefore, the specificity of these findings in terms of neuroanatomy, timing, and retinotopic location is consistent with the hypothesis that foveal cortex is a crucial recipient of late feedback signals from the visual periphery.

The present results raise a number of questions about the content and functionality of foveal feedback. Existing fMRI evidence indicates that representations of the visual periphery in foveal cortex contain only task-relevant information (Williams, et al., 2008), encoding the behaviourally relevant shape, but not the irrelevant colour, of peripheral objects. This evidence, combined with the current TMS results, suggests that constructive feedback reflects more than a mere artefact of visual processing or the point spread of the haemodynamic response (Arthurs and Boniface, 2003; Logothetis, 2008). Instead, constructive feedback appears to be an instrumental component of visual perception, required whenever task goals require covert allocation of attention to objects in peripheral space.

Since our behavioural task required covert selection of the visual periphery, might our TMS results be explained by the same feedback mechanisms that are known to support attention? (Desimone and Duncan, 1995; Duncan, et al., 1997; Kastner and Ungerleider, 2000; Pessoa et 
al., 2003). Conventional accounts of feedback, including those implicated in attention, involve modulation of existing feedforward representations; however our data indicate that $\mathrm{p}$ calcarine TMS did not interfere significantly with early feedforward processing of peripheral stimuli. Furthermore, the critical epoch we observed in primary visual cortex $(+350-400 \mathrm{msec})$ is $\sim 100 \mathrm{msec}$ later than would be expected solely by re-entrant attentional modulation (Juan and Walsh, 2003; Martinez, 1999; Martinez et al., 2001; Noesselt et al., 2002) and raises the possibility that foveal feedback, while likely dependent on goal-directed attention, may incur additional computational costs. Consistent with previous explanations of late occipital feedback (Heinen et al. 2005), this computation could potentially relate to perceptual decision-making processes, implemented by a constructive feedback mechanism that exploits the fine-grained spatial resolution of foveal cortex to 'rebuild' and discriminate between extra-foveal representations. Recent evidence raises the possibility that this top-down process may be linked to visual working memory (Harrison and Tong, 2009; Serences et al., 2009) and mental imagery (Stokes et al., 2009). Within either of these domains, foveal visual cortex may serve as a dynamic workspace, or 'scratchpad', for increasing the precision of perceptual decisions (Baars, 1988). Interestingly, late TMS in Experiment 2 did not reliably impair discrimination of foveal stimuli (Figure 3a). While this null effect should be treated with caution, it nevertheless suggests that this 'scratchpad' may be especially important for resolving extra-foveal representations, perhaps in advance of a goal-directed saccade that would foveate the stimulus under naturalistic conditions.

The existence of foveal feedback implies the actions of a key source (or sources) that transmit behaviourally relevant information to the foveal cortex. Key candidates include the lateral occipital complex (LOC: Pitcher et al., 2009), extrastriate cortex (Pascual-Leone and Walsh, 2001; Silvanto et al., 2005b) as well as parietal and frontal areas (Chambers and Mattingley, 2005; Corbetta et al., 2008; Stokes, et al., 2009). The ability to measure foveal feedback with fMRI, and now with TMS, opens a number of promising avenues for combining these methods in future studies, for instance, by determining the effect of LOC stimulation on peripheral information encoded in foveal V1. More broadly, further studies of the mechanism by which top-down sources modulate striate cortex (Ruff, et al., 2006; Silvanto et al., 2009) are likely to have important implications for understanding the nature of foveal feedback in visual information processing. 


\section{Acknowledgments}

This research was supported by the Biotechnology and Biological Sciences Research Council (CDC, David Phillips Fellowship, UK), the Australian Research Council (MAW, Queen Elizabeth II Fellowship), the Wales Institute of Cognitive Neuroscience (CDC) and a Cardiff University International Collaborative Award (CDC/MAW). We thank Martynas Dervinis for technical assistance, and Chris Baker, Hans Op de Beeck, Nancy Kanwisher, and Anina Rich for helpful discussions.

\section{References}

Aickin M, and Gensler H. Adjusting for multiple testing when reporting research results: The Bonferroni vs. Holm methods. American Journal of Public Health, 86(5): 726, 1996.

Amassian VE, Cracco RQ, Maccabee PJ, Cracco JB, Rudell A, Eberle L. Suppression of visual perception by magnetic coil stimulation of human occipital cortex. Electroencephalography and Clinical Neurophysiology, 74(6):458-462, 1989.

Arthurs OJ, and Boniface SJ. What aspect of the fMRI BOLD signal best reflects the underlying electrophysiology in human somatosensory cortex? Clinical Neurophysiology, 114(7): 1203-1209, 2003.

Baars BJ. A Cognitive Theory of Consciousness. Cambridge, MA: Cambridge University Press, 1988.

Camprodon JA, Zohary E, Brodbeck V, and Pascual-Leone A. Two phases of V1 activity for visual recognition of natural images. Journal of Cognitive Neuroscience, 22(6): 1262 1269, 2010.

Chambers CD, and Mattingley JB. Neurodisruption of selective attention: insights and implications. Trends in Cognitive Sciences, 9(11): 542, 2005.

Corbetta M, Patel G, and Shulman GL. The reorienting system of the human brain: From environment to theory of mind. Neuron, 58(3): 306-324, 2008.

Corthout E, Uttl B, Ziemann U, Cowey A, and Hallet M. Two periods of processing in the (circum)striate visual cortex as revealed by transcranial magnetic stimulation. Neuropsychologia, 37(2): 137-145, 1999.

de Graaf TA, Herring J, and Sack, AT. A chronometric exploration of high-resolution 'sensitive TMS masking' effects on subjective and objective measures of vision. Experimental Brain Research, 209(1):19-27, 2011.

Desimone R, and Duncan J. Neural mechanisms of selective visual attention. Annual Review of Neuroscience, 18: 193-222, 1995.

Duncan J, Humphreys G, and Ward R. Competitive brain activity in visual attention. Current Opinion in Neurobiology, 7(2): 255-261, 1997.

Duncan RO, and Boynton, GM. Cortical magnification within human primary visual cortex correlates with acuity thresholds. Neuron, 38(4): 659-671, 2003.

Gilbert CD. Circuitry, architecture, and functional dynamics of visual cortex. Cerebral Cortex, 3(5): 373-386, 1993.

Harrison S, and Tong F. Decoding reveals the contents of visual working memory in early visual areas. Nature, 458(7238): 632-635, 2009. 
Heinen K, Jolij J, and Lamme VA. Figure-ground segregation requires two distinct periods of activity in V1: a transcranial magnetic stimulation study. NeuroReport, 16(13): 14831487, 2005.

Juan $\mathrm{CH}$, and Walsh V. Feedback to V1: a reverse hierarchy in vision. Experimental Brain Research, 150(2): 259-263, 2003.

Kammer, T, Beck, S, Erb, M, and Grobb, W. The influence of current direction on phosphene thresholds evoked by transcranial magnetic stimulation. Clinical Neurophysiology, 112(11): 2015-2021, 2001.

Kammer T, Puls K, Strasburger H, Hill N, and Wichmann F. Transcranial magnetic stimulation in the visual system. I. The psychophysics of visual suppression. Experimental Brain Research, 160(1): 118-128, 2005.

Kastner S, and Ungerleider L. Mechanisms of visual attention in the human cortex. Annual Review of Neuroscience, 23: 315-341, 2000.

Koivisto M, Mäntylä T, and Silvanto, J. The role of early visual cortex (V1/V2) in conscious and unconscious visual perception. Neuroimage, 51(2): 828-834, 2010.

Kosslyn S, Ganis G, and Thompson W. Neural foundations of imagery. Nature Reviews Neuroscience, 2(9): 635-642, 2001.

Kosslyn SM, Pascual-Leone A, Felician O, Camposano S, Keenan JP, Thompson WL, Ganis G, Sukel KE, and Alpert NM. The role of Area 17 in visual imagery: convergent evidence from PET and rTMS. Science, 284(5411): 167-170, 1999.

Lamme V, and Roelfsema PR. The distinct modes of vision offered by feedforward and recurrent processing. Trends in Neuroscience, 23(11): 571-579, 2000.

Lamme V, Supèr H, and Spekreijse H. Feedforward, horizontal, and feedback processing in the visual cortex. Current Opinion in Neurobiology, 8(4): 529-535, 1998.

Layock R, Crewther DP, Fitzgerald PG, and Crewther SG. Evidence for fast signals and later rrocessing in human V1/V2 and V5/MT+: A TMS study of motion perception. Journal of Neurophysiology, 98(3): 1253-1262, 2007.

Logothetis NK. What we can do and what we cannot do with fMRI. Nature, 453(7197): 869878, 2008.

Martinez A. Involvement of striate and extrastriate visual cortical areas in spatial attention. Nature Neuroscience, 2(4): 364-369, 1999.

Martinez A, Di Russo F, Anllo-Vento L, and Hillyard S. Electrophysiological analysis of cortical mechanisms of selective attention to high and low spatial frequencies. Clinical Neurophysiology, 112(11): 1980-1998, 2001.

Müller HJ, and Rabbitt PMA. Reflexive and voluntary orienting of visual-attention - time course of activation and resistance to interruption. Journal of Experimental Psychology-Human Perception and Performance, 15(2): 315-330, 1989.

Noesselt T, Hillyard SA, Woldorff MG, Schoenfeld A, Hagner T, Jancke L, Tempelmann C, Hinrichs $\mathrm{H}$, and Heinze HJ. Delayed striate cortical activation during spatial attention. Neuron, 35(3): 575-587, 2002.

Op de Beeck H, Baker C, DiCarlo J, and Kanwisher N. Discrimination training alters object representations in human extrastriate cortex. Journal of Neuroscience, 26(50): 1302513036, 2006.

Pascual-Leone A, and Walsh A. Fast backprojections from the motion to the primary visual area necessary for visual awareness. Science, 292(5516): 510-512, 2001.

Pessoa L, Kastner S, and Ungerleider L. Neuroimaging studies of attention: From modulation of sensory processing to top-down control. The Journal of Neuroscience, 23(10): 3990-3998, 2003.

Pitcher D, Charles L, Devlin JT, Walsh V, and Duchaine B. Triple dissociation of faces, bodies, and objects in extrastriate cortex. Current Biology, 19(4): 319-324, 2009. 
Ress D, and Heeger DJ. Neuronal correlates of perception in early visual cortex. Nature Neuroscience, 6(4): 414-420, 2003.

Ro T, Breitmeyer B, Burton P, Singhal NS, and Lane D. Feedback contributions to visual awareness in human occipital cortex. Current Biology, 13(12): 1038-1041, 2003.

Rorden C, and Brett M. Stereotaxic display of brain lesions. Behavioural Neurology, 12(4): 191-200, 2000.

Ruff CC, Blankenburg F, Bjoertomt O, Bestmann S, Freeman E, Haynes J-D, Rees G, Josephs O, Deichmann R, and Driver J. Concurrent TMS-fMRI and psychophysics reveal frontal influences on human retinotopic visual cortex. Current Biology, 16(15): 1479-1488, 2006.

Sawaki L, Okita T, Fujiwara M, and Mizuno K. Specific and non-specific effects of transcranial magnetic stimulation on simple and go/no-go reaction time. Experimental Brain Research, 127(4): 402-408, 1999.

Schenkluhn BM, Ruff CC, Heinen K, and Chambers CD. Parietal stimulation decouples spatial and feature-based attention. Journal of Neuroscience, 28(44): 11106-11110, 2008.

Serences J, Ester E, Vogel E, and Awh E. Stimulus-specific delay activity in human primary visual cortex. Psychological Science, 20(2): 207-214, 2009.

Silvanto J, Lavie N, and Walsh V. Double dissociation of V1 and V5/MT activity in visual awareness. Cerebral Cortex, 15(11): 1736-1741, 2005 a.

Silvanto J, Cowey A, Lavie N, and Walsh V. Striate cortex (V1) activity gates awareness of motion. Nature Neuroscience, 8(2): 143-144, 2005 b.

Silvanto J, Muggleton NG, Lavie N, and Walsh A. The perceptual and functional consequences of parietal top-down modulation on the visual cortex. Cerebral Cortex, 19(2): 327-330, 2009.

Stevens LK, McGraw PV, Ledgeway T, and Schluppeck D. Temporal characteristics of global motion processing revealed by transcranial magnetic stimulation. European Journal of Neuroscience, 30(12): 2415-2426, 2009.

Stokes MG, Thompson R, Cusack R, and Duncan J. Top-Down activation of shape-specific population codes in visual cortex during mental imagery. Journal of Neuroscience, 29(5): 1565-1572, 2009.

Super H, Spekreijse H, and Lamme V. Two distinct modes of sensory processing observed in monkey primary visual cortex (V1). Nature Neuroscience, 4(3): 304-310, 2001.

Varnava, A, Stokes, MG, \& Chambers, CD (2011). Reliability of the 'observation of movement' method for determining motor threshold using transcranial magnetic stimulation. Journal of Neuroscience Methods, 201(2): 327-332, 2011.

Williams M, Baker C, Op de Beeck H, Shim W, Dang S, Triantafyllou C, and Kanwisher N. Feedback of visual object information to foveal retinotopic cortex. Nature Neuroscience, 11(12): 1439-1445, 2008. 
Table 1. Mean and SEM of coefficients resulting from the Gaussian non-linear regressions. Only high-intensity TMS of the calcarine site during the late phase significantly reduced discrimination accuracy. ${ }^{*} p<0.05$ following Holm-Bonferroni correction for twelve comparisons (strictest $\alpha=.00416$ ).

\begin{tabular}{|c|c|c|c|c|c|c|c|}
\hline TMS & Site & $R^{2}$ & $y_{0}$ & Period & $\begin{array}{c}\text { Peak Amp } \\
\left(a_{1} \mid a_{2}\right) \Delta d^{\prime}\end{array}$ & $\begin{array}{c}\text { Peak Time } \\
\left(x_{1} \mid x_{2}\right) \mathrm{msec}\end{array}$ & $\begin{array}{c}\text { Bandwidth } \\
\left(b_{1} \mid b_{2}\right) \text { msec }\end{array}$ \\
\hline \multirow{6}{*}{ High } & \multirow{2}{*}{ Calcarine } & \multirow{2}{*}{$0.83(0.04)$} & \multirow{2}{*}{$1.44(0.08)$} & $1^{\mathrm{st}}$ & $-0.05(0.33)$ & N/A & N/A \\
\hline & & & & $2^{\text {nd }}$ & $-0.69(0.20)^{*}$ & $332.2(20.5)$ & $59.8(16.5)$ \\
\hline & \multirow{2}{*}{ Non-calc } & \multirow{2}{*}{$0.88(0.02)$} & \multirow{2}{*}{$1.40(0.09)$} & $1^{\text {st }}$ & $0.49(0.38)$ & N/A & $\mathrm{N} / \mathrm{A}$ \\
\hline & & & & $2^{\text {nd }}$ & $0.21(0.30)$ & N/A & N/A \\
\hline & \multirow{2}{*}{ Sham } & \multirow{2}{*}{$0.90(0.02)$} & \multirow{2}{*}{$1.27(0.11)$} & $1^{\mathrm{st}}$ & $0.02(0.23)$ & $\mathrm{N} / \mathrm{A}$ & N/A \\
\hline & & & & $2^{\text {nd }}$ & $0.02(0.24)$ & N/A & N/A \\
\hline \multirow{6}{*}{ Low } & \multirow{2}{*}{ Calcarine } & \multirow{2}{*}{$0.91(0.02)$} & \multirow{2}{*}{$1.23(0.09)$} & $1^{\mathrm{st}}$ & $0.43(0.37)$ & N/A & $\mathrm{N} / \mathrm{A}$ \\
\hline & & & & $2^{\text {nd }}$ & $0.15(0.25)$ & N/A & $\mathrm{N} / \mathrm{A}$ \\
\hline & \multirow{2}{*}{ Non-calc } & \multirow{2}{*}{$0.83(0.03)$} & \multirow{2}{*}{$1.37(0.10)$} & $1^{\mathrm{st}}$ & $0.42(0.26)$ & N/A & $\mathrm{N} / \mathrm{A}$ \\
\hline & & & & $2^{\text {nd }}$ & $0.29(0.33)$ & N/A & N/A \\
\hline & \multirow{2}{*}{ Sham } & \multirow{2}{*}{$0.92(0.02)$} & \multirow{2}{*}{$1.18(0.13)$} & $1^{\mathrm{st}}$ & $0.64(0.39)$ & N/A & $\mathrm{N} / \mathrm{A}$ \\
\hline & & & & $2^{\text {nd }}$ & $-0.07(0.37)$ & N/A & $\mathrm{N} / \mathrm{A}$ \\
\hline
\end{tabular}




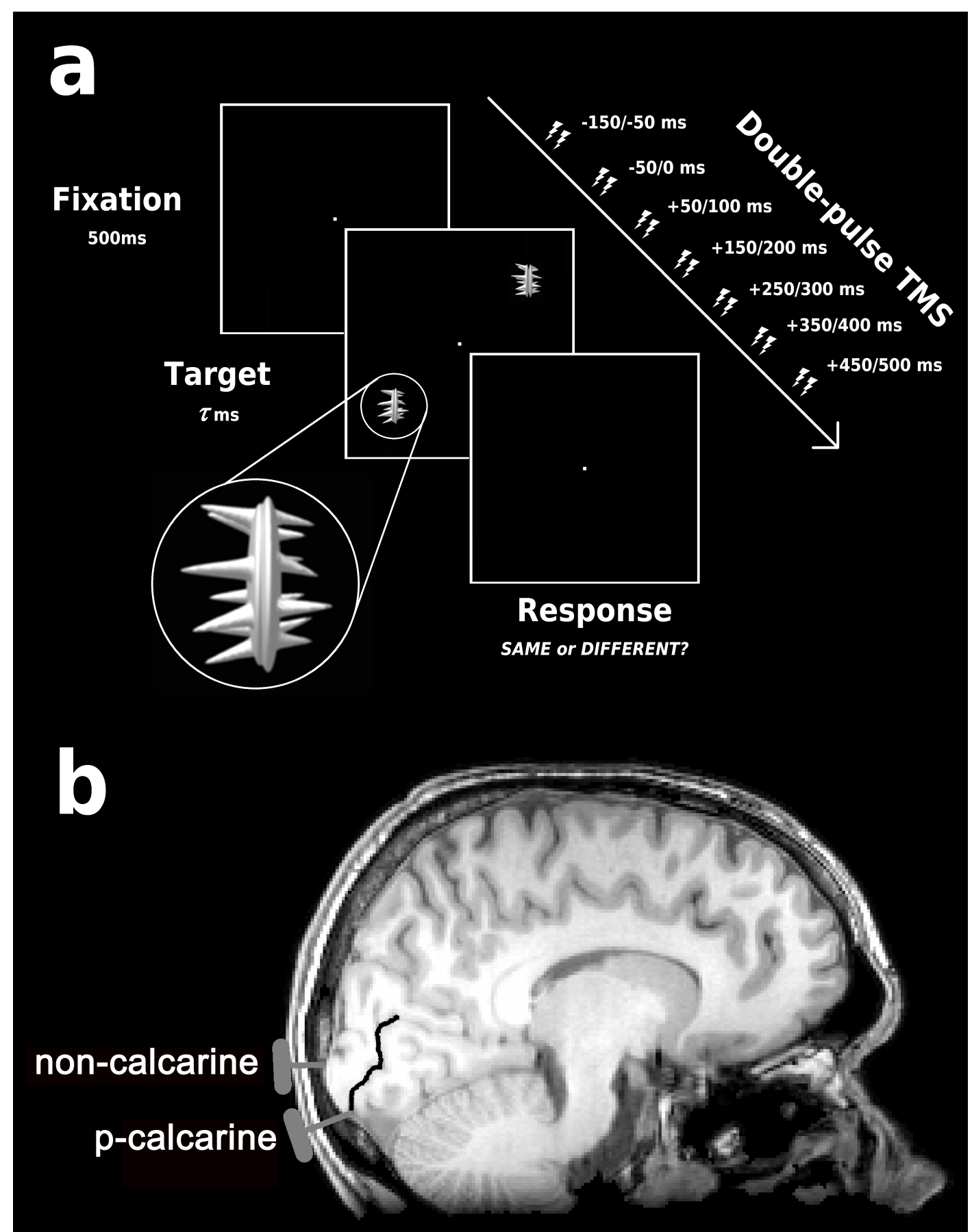

Figure 1. Experimental paradigm and sites of cortical stimulation. (a) Schematic of visual displays in Experiment 1. The target array consisted of two objects presented in opposite diagonal locations, drawn randomly from a set of 'spiky' stimuli (Williams, et al., 2008); $7^{\circ}$ eccentricity; diagonal configuration alternated between blocks). On each trial, participants decided whether the two objects were the same or different. Prior to the TMS experiment, the duration of the target array $(\tau)$ was adjusted on an individual basis to yield $70-75 \%$ correct discriminations. In Experiment 2, the task and stimulus sequence was broadly identical with the exception that stimuli were presented either on the fovea or in the periphery, with TMS was applied to the p-calcarine site at either $+50 / 100$ or $+350 / 400$ msec. (b) TMS sites in one participant. The p-calcarine site (foveal representation) was defined as the posterior termination of the calcarine sulcus (black line). The non-calcarine control site was localised $\sim 15 \mathrm{~mm}$ rostral to the calcarine site. All regions were localised within individual MRI scans. 
(a)

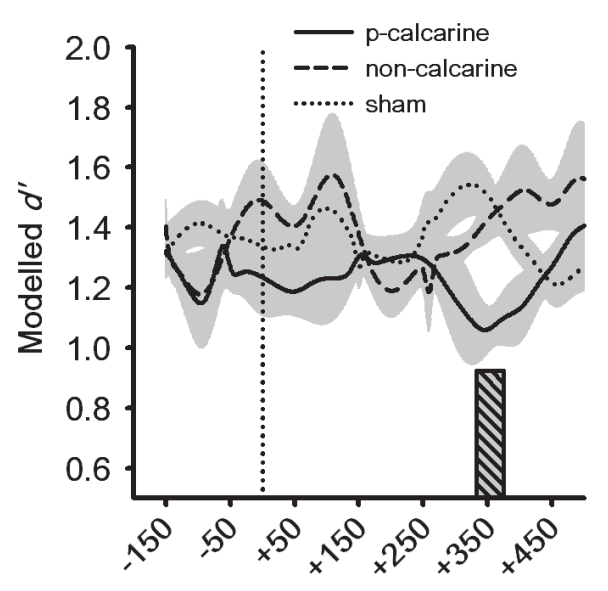

(b)

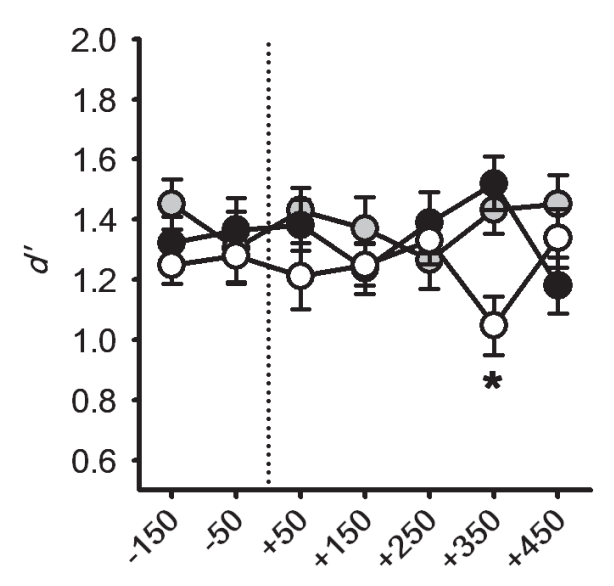

TMS Onset (msec)

TMS Onset (msec)

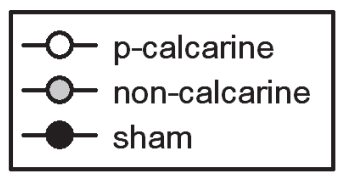

(c)

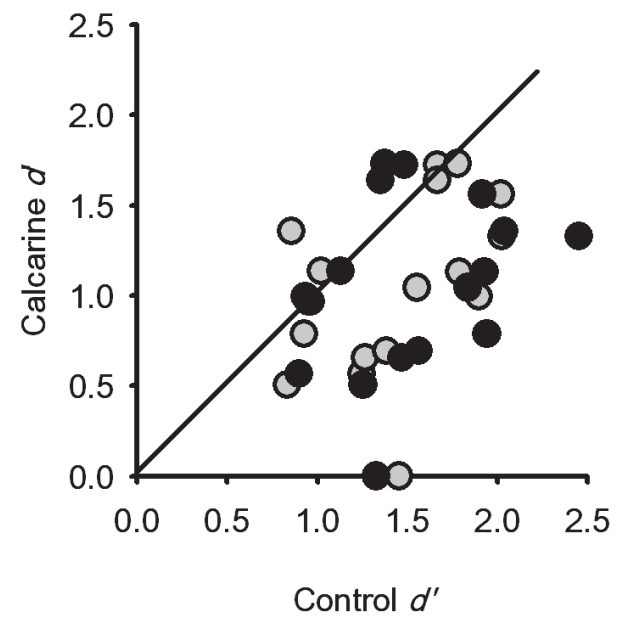

(d)

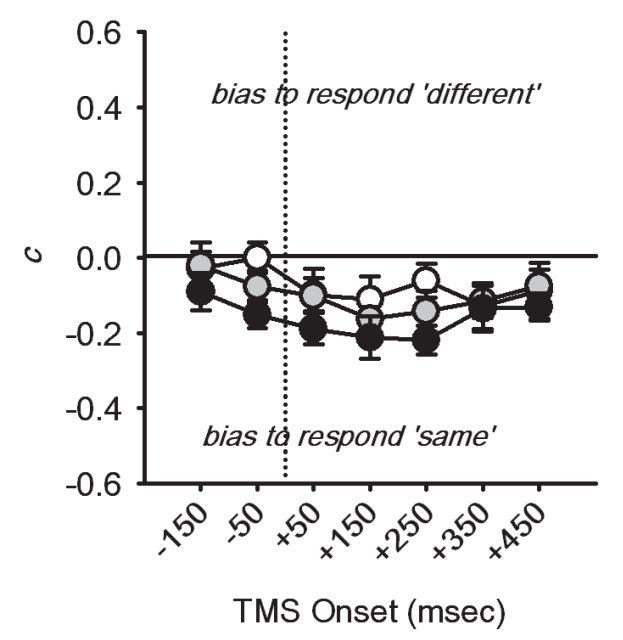

Figure 2. The effect of occipital TMS on perception of peripheral stimuli in Experiment 1. (a) Average double-Gaussian models of $d^{\prime}$ during high-intensity TMS in the p-calcarine, non-calcarine and sham conditions. The grey shaded regions indicate \pm 1 SE of the average model at each TMS onset time. The hatched bar indicates the range of TMS onset times at which the average modelled $d^{\prime}$, during p-calcarine TMS, differed significantly from both the non-calcarine and sham conditions (by paired-samples $t$-test with Holm-Bonferroni corrections). See Table 1 for average Gaussian coefficients. (b) Average $d^{\prime}$ for high-intensity TMS trials as a function of TMS onset time and TMS site. Consistent with the non-linear analysis, TMS at a relatively late onset time $(+350 / 400 \mathrm{msec})$ impaired discrimination accuracy $(*)$. (c) Individual results at the TMS onset time of $+350 / 400 \mathrm{msec}$. Data points falling to the right of the unity line indicate a reduction in $d^{\prime}$ caused by calcarine stimulation. (d) Average response bias for high-intensity TMS trials according to TMS onset time and TMS site. Overall, TMS of the p-calcarine and non-calcarine sites reduced the tendency to respond 'same' (relative to sham); however this general effect did not interact with TMS onset time. Error bars in all panels are \pm 1 SEM. 
(a) Foveal

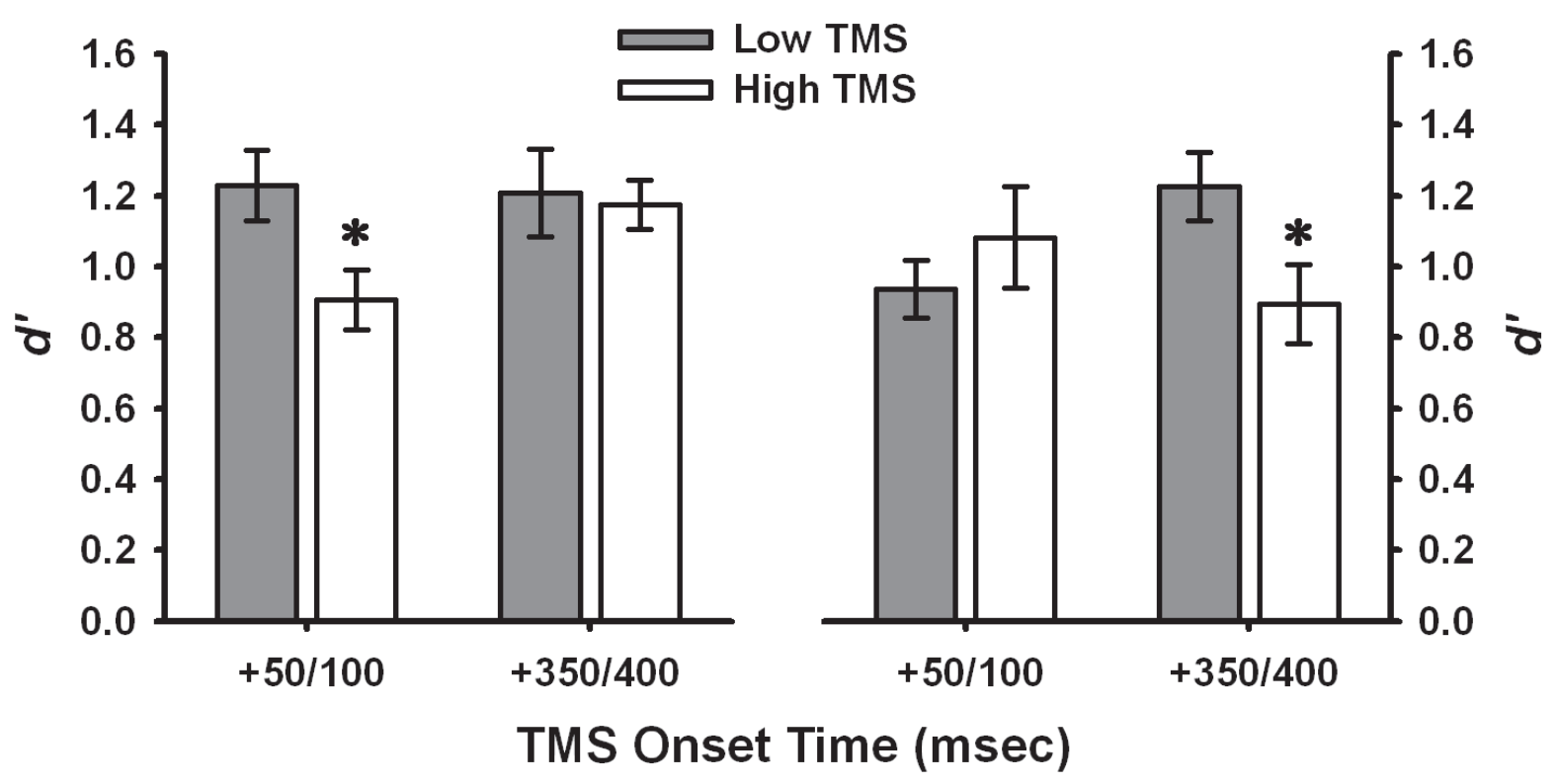

Figure 3. The effect of p-calcarine stimulation on foveal and peripheral perception in Experiment 2. (a) Average $d^{\prime}$ for foveal stimuli plotted according to TMS intensity and TMS onset time. Only early TMS (+50/100ms) impaired performance. (b) Average $d^{\prime}$ for peripheral stimuli plotted according to TMS intensity and TMS onset time. Only late TMS $(+350 / 400 \mathrm{~ms})$ impaired performance. Error bars are $\pm 1 \mathrm{SE}$ of the mean. $* p<.05$ for comparison of low- $v$ s. high-intensity TMS. 